\title{
Multispacer Sequence Typing Relapsing Fever Borreliae in Africa
}

\author{
Elbir Haitham ${ }^{1}$, Gregory Gimenez ${ }^{1}$, Cheikh Sokhna ${ }^{2}$, Kassahun Desalegn Bilcha ${ }^{3}$, Jemal Ali ${ }^{3}$, \\ Stephen C. Barker ${ }^{4}$, Sally J. Cutler ${ }^{5}$, Didier Raoult ${ }^{1}$, Michel Drancourt ${ }^{1 *}$
}

1 Unité de Recherche sur les Maladies Infectieuses et Tropicales Emergentes (URMITE), UMR CNRS 6236 IRD 198, Faculté de Médecine, Méditerranée Infection, Aix-Marseille Université, Marseille, France, 2 Unité de Recherche sur les Maladies Infectieuses et Tropicales Emergentes (URMITE), UMR CNRS 6236 IRD 198 , Méditerranée Infection, AixMarseille Université, Dakar, Senegal, $\mathbf{3}$ College of Medicine and Health Sciences, University of Gondar, Gondar, Ethiopia, 4 Parasitology Section, School of Chemistry and Molecular Bioscience, University of Queensland, Brisbane, Australia, $\mathbf{5}$ School of Health, Sports and Bioscience, University of East London, London, United Kingdom

\begin{abstract}
Background: In Africa, relapsing fevers are neglected arthropod-borne infections caused by closely related Borrelia species. They cause mild to deadly undifferentiated fever particularly severe in pregnant women. Lack of a tool to genotype these Borrelia organisms limits knowledge regarding their reservoirs and their epidemiology.

Methodology/Principal Findings: Genome sequence analysis of Borrelia crocidurae, Borrelia duttonii and Borrelia recurrentis yielded 5 intergenic spacers scattered between 10 chromosomal genes that were incorporated into a multispacer sequence typing (MST) approach. Sequencing these spacers directly from human blood specimens previously found to be infected by B. recurrentis (30 specimens), B. duttonii (17 specimens) and B. crocidurae (13 specimens) resolved these 60 strains and the 3 type strains into 13 species-specific spacer types in the presence of negative controls. $B$. crocidurae comprised of 8 spacer types, B. duttonii of 3 spacer types and B. recurrentis of 2 spacer types.

Conclusions/Significance: Phylogenetic analyses of MST data suggested that $B$. duttonii, $B$. crocidurae and B. recurrentis are variants of a unique ancestral Borrelia species. MST proved to be a suitable approach for identifying and genotyping relapsing fever borreliae in Africa. It could be applied to both vectors and clinical specimens.
\end{abstract}

Citation: Haitham E, Gimenez G, Sokhna C, Bilcha KD, Ali J, et al. (2012) Multispacer Sequence Typing Relapsing Fever Borreliae in Africa. PLoS Negl Trop Dis 6(6): e1652. doi:10.1371/journal.pntd.0001652

Editor: Joseph M. Vinetz, University of California San Diego School of Medicine, United States of America

Received February 10, 2012; Accepted April 9, 2012; Published June 5, 2012

Copyright: (C) 2012 Haitham et al. This is an open-access article distributed under the terms of the Creative Commons Attribution License, which permits unrestricted use, distribution, and reproduction in any medium, provided the original author and source are credited.

Funding: The authors acknowledge the financial support of ANR 2008 BORETIC. The funders had no role in study design, data collection and analysis, decision to publish, or preparation of the manuscript.

Competing Interests: The authors have declared that no competing interests exist.

* E-mail: Michel.Drancourt@univmed.fr

\section{Introduction}

In Africa, relapsing fevers (RF) are arthropod-borne diseases caused by four cultured species Borrelia crocidurae, Borrelia duttonii, Borrelia hispanica and Borrelia recurrentis [1]. Transmission is by the bite of Ornithodoros soft ticks for the first three species whereas Pediculus humanus louse feces transmit $B$. recurrentis [2,3]. In Tanzania, molecular investigations of human and tick specimens further provided evidences for two additional, yet uncultured Borrelia species [1,4]. Each one of the four cultured Borrelia species is more prevalent in one geographical area of Africa with $B$. hispanica being reported in Morocco [5], B. crocidurae in Senegal [6], B. duttonii in Tanzania [7] and B. recurrentis in Ethiopia [8]. However, the precise area of distribution of each Borrelia is unknown and may overlap as both $B$. duttonii and B. crocidurae have been reported in Togo and Tanzania $[1,9]$.

In these regions of Africa, RF was reported to be the most prevalent bacterial disease, accounting for $8.8 \%$ of febrile patients in Togo [9]. In Senegal, average incidence is 11 per 100 personyears [10]. The main clinical symptom of infection is recurrent undifferentiated fever associated with high bacteremia; RF are therefore often diagnosed as malaria and cases of malaria co- infection with have been reported $[9,11,12]$. RF are treatable by antibiotics. Severity ranges from asymptomatic to fatal, particularly if left untreated and can be associated with significant pregnancy loss or peri-natal mortality $[13,14,15]$.

The African RF Borrelia are very closely related species as illustrated by $16 \mathrm{~S}$ rRNA gene sequence variability $\leq 1 \%$ [2]. Accordingly, a previous comparison of $B$. duttonii and B. recurrentis genomes indicated that the two organisms formed a unique bacterial species [16]. Such a close genetic and genomic proximity challenged the development of laboratory tools for the accurate discrimination between the African RF Borrelia and genotyping [16]. Sequencing the $16 \mathrm{~S}$ rRNA and the flagellin genes is unsatisfactory since African RF Borrelia differ by only one base in the flagllin gene sequence and have 16S rRNA gene sequence similarity above 99\% [17]. Analysis of the intrergenic spacer (IGS) located between the $16 \mathrm{~S}$ and $23 \mathrm{~S}$ rRNA genes only explored the variability between $B$. duttonii and B. recurrentis [1]. Moreover, IGS sequence overlapped between one $B$. duttonii phylogenetic group and one $B$. recurrentis group [1] with a second overlap disclosed with subsequent analyses of further material [7].

We previously observed that multispacer sequence typing (MST), a PCR-sequencing-based method for bacteria genotyping, 


\section{Author Summary}

In Africa, relapsing fevers are caused by four cultured species: Borrelia crocidurae, Borrelia duttonii, Borrelia hispanica and Borrelia recurrentis. These borreliae are transmitted by the bite of Ornithodoros soft ticks except for $B$. recurrentis which is transmitted by louse Pediculus humanus. They cause potentially undifferentiated fever infection and co-infection with malaria could also occur. The exact prevalence of each Borrelia is unknown and overlaps between $B$. duttonii and $B$. crocidurae have been reported. The lack of tools for genotyping these borreliae limits knowledge concerning their epidemiology. We developed multispacer sequence typing (MST) and applied it to blood specimens infected by $B$. recurrentis (30 specimens), $B$. duttonii (18 specimens) and $B$. crocidurae (13 specimens), delineating these 60 strains and the 3 type strains into 13 species-specific spacer types. $B$. crocidurae strains were classified into 8 spacer types, B. duttonii into 3 spacer types and $B$. recurrentis into 2 spacer types. These findings provide the proof-of-concept that that MST is a reliable tool for identification and genotyping relapsing fever borreliae in Africa.

was efficient in typing otherwise homogenous bacterial species such as the plague agent Yersinia pestis [18] and the typhus agent Rickettsia prowazekii [19]. Ongoing study of the B. crocidurae genome in our laboratory gave us the opportunity to develop MST for African RF Borrelia and to deliver the proof-of-concept that MST is a suitable method for both the species identification and genotyping of RF Borrelia in Africa.

\section{Materials and Methods}

\section{Borrelia strains and DNA}

B. crocidurae Achema strain, B. recurrentis Al strain and B. duttonii Ly strain were grown in BSK-H medium (Sigma, Saint Quentin Fallavier, France) supplemented with heat-inactivated 10\% rabbit serum (Eurobio, Courtaboeuf, France). B. recurrentis DNA was extracted from 21 blood specimens collected in 1994 in Addis Ababa, Ethiopia Dr. S. J. Cutler (School of Health, Sports and Bioscience, University of East London, London UK). Likewise, $B$. recurrentis DNA extracted from 9 blood specimens collected in 2011 in Bahir Dah, Highlands of Ethiopia were provided by SC Barker (Parasitology section, School of Chemistry and Molecular Biosci- ence, University of Queensland, Brisbane, Australia) and KD Bilcha and J Ali (University of Gondar, Ethiopia). In addition, $B$. duttonii DNA extracted from $\mathbf{1 7}$ blood specimens collected in Mvumi, Tanzania were also provided by Dr. S. J. Cutler. B. crocidurae DNA was extracted from 13 blood specimens collected in 2010 in Senegal by C. Sokhna (URMITE, Dakar, Senegal) including 11 specimens from Dielmo and 2 specimens from Ndiop. DNA was extracted from these specimens using QIAamp DNA Blood mini kits (QIAGEN, Hilden, Germany) according to the manufacturer's instructions.

\section{Selection of intergenic spacers}

The B. crocidurae genome (Genbank accession number CP003426CP003465) has been sequenced and annotated in our laboratory using pyrosequencing technology on a Roche 454 GS FLX sequencer. The draft genome is comprising of one closed chromosome and scaffolds representing the plasmids. Spacer sequences extracted from B. crocidurae strain Achema, B. recurrentis strain Al (Genbank accession number CP000993) and B. duttonii strain Ly (Genbank accession number CP000976) genomes using perl script software were compared using ssaha2 software [20]. Spacers were pre-selected for a 300 to 800 -bp length. Pre-selected spacers were further analyzed for sequence similarity in order to exclude spacers with $<0.1 \%$ interspecies sequence similarity. PCR primers were then designed using primer3 software (http://fokker.wi.mit.edu) in order to amplify the entire sequence of each of the selected spacers.

\section{Multispacer sequencing typing}

Five microliters of Borrelia DNA and $10 \mathrm{pmol}$ of each primer (Eurogentec, Seraing, Belgium) were added to the PCR mixture, containing 0.4 U Phusion DNA Polymerase (Finnzymes, Espoo, Finland), $4 \mu \mathrm{l}$ of $5 \times$ Phusion HF Buffer (Finnzymes) and $0.4 \mu \mathrm{l}$ of $10 \mathrm{mM}$ dNTPs. The volume was adjusted to $24 \mu \mathrm{L}$ by adding distilled water. Thermal cycling was performed on a 2720 DNA thermal cycler (Applied Biosystems, Courtaboeuf, France) with an initial 30 -sec cycle at $98^{\circ} \mathrm{C}$ followed by 35 cycles consisting of 10 seconds at $98^{\circ} \mathrm{C}, 30$ seconds at $58^{\circ} \mathrm{C}$ and 1 minute at $72^{\circ} \mathrm{C}$, followed by a 10 -min final extension step at $72^{\circ} \mathrm{C}$. To rule out amplicon carry-over, nucleotide-free water negative control was used throughout the steps of the protocol. PGR products were purified prior to sequencing by using the Nucleo-Fast 96 PGR Kit (Macherey-Nagel, Hoerdt, France). Three microliters of the resulting DNA were added to each primer mixture comprised of 10 pmol of each primer, $4 \mu \mathrm{L}$ water and $3 \mu \mathrm{L}$ BigDye Terminator reaction mix (Applied Biosystems). Sequencing thermal cycling

Table 1. List of primers and genes flanking five intergenic spacers herein studied in relapsing fever Borrelia.

\begin{tabular}{|c|c|c|c|c|c|}
\hline Spacers & Start End & Spacer flanking genes (5-----3) & Primers & PCR product size (bp) & Spacer size (bp) \\
\hline MST2 & $786480 . .786968$ & $\begin{array}{l}\text { penicillin-binding protein//uncharacterized } \\
\text { conserved protein }\end{array}$ & $\begin{array}{l}\text { F:TTTTGCTAAAATTAACCCTTTTCA } \\
\text { R:CTCATTTTAATTCCTTACCCCTA }\end{array}$ & 578 & 487 \\
\hline MST3 & 669736.. 670279 & $\begin{array}{l}\mathrm{N} \text {-acetylmuramoyl-L-alanine amidase, } \\
\text { putative//vacuolar X-prolyl dipeptidyl } \\
\text { aminopeptidase I }\end{array}$ & $\begin{array}{l}\text { F:GCAGGTGGCTGTTAACCACT } \\
\text { R:ATGTGGGGAATGCACTCTIT }\end{array}$ & 687 & 543 \\
\hline MST5 & 565860.. 566397 & $\begin{array}{l}\text { translation elongation factor } \mathrm{G} / / \\
\text { uncharacterized conserved protein }\end{array}$ & $\begin{array}{l}\text { F:CCTGAGTCGATATGGGCACT } \\
\text { R:CAACCTGACATATCTTACTCAATTCAT }\end{array}$ & 653 & 536 \\
\hline MST6 & 494656.. 494903 & $\begin{array}{l}\text { tRNA-ser//DNA polymerase III subunits } \\
\text { gamma and tau }\end{array}$ & $\begin{array}{l}\text { F:GGGTTCGAATCCCATTTTCT } \\
\text { R:CTCTGGGACGCCTCTTAATG }\end{array}$ & 333 & 246 \\
\hline MST7 & 458283.. 458778 & $16 \mathrm{~S}$ ribosomal RNA//hypothetical protein & $\begin{array}{l}\text { F:TTCGCCACTGAATGTATTGC } \\
\text { R:TGCCAATGTCTTGTGGTC }\end{array}$ & 738 & 494 \\
\hline
\end{tabular}

Start and end of spacer are according to B. duttonii genome.

doi:10.1371/journal.pntd.0001652.t001 
was performed on a Applied Biosystems DNA thermal cycler with an initial 5-min cycle at $96^{\circ} \mathrm{C}$ followed by 25 cycles consisting of 30 seconds at $96^{\circ} \mathrm{C}, 20$ seconds at $55^{\circ} \mathrm{C}$, and 4 minutes at $60^{\circ} \mathrm{C}$, followed by a 7 -min final extension step at $15^{\circ} \mathrm{C}$. Sequencing products were purified using sephadex plates (Sigma-Aldrich, Saint Quentin Fallavier, France) and sequencing electrophoresis was performed on a 3130 Genetic Analyzer (Applied Biosystems).

\section{Sequence analysis}

The nucleotide sequences were edited using ChromasPro software (www.technelysium.com.au/chromas.html). Similarities between spacers were determined after multiple alignments using the MULTALIN software [21]. MST discrimination power was calculated using the Hunter-Gaston Index [22]:

$$
D I=1-\left[\frac{1}{N(N-1)} \sum_{j-1}^{8} n_{j}\left(n_{j}-1\right)\right]
$$

where $D$ is the numerical index of discrimination, $\mathcal{N}$ is the total number of isolates in the sample population, $s$ is the total number of different types, and $n j$ is the number of isolates belonging to the $j$ th type.

The five spacer sequences analyzed herein were concatenated and neighbor-joining phylogenetic tree was reconstructed using the maximum likelihood method in PhyML 3.0 [23]. Each particular sequence of a given spacer was assigned to a spacer type (ST) number.

\section{Ethics statement}

This study was approved by the IFR48 Ethic Committee. All patients provided informed written consent.

\section{Results}

\section{Spacer selection}

Chromosome sequence alignment of the three Borrelia reference genomes studied herein revealed that 23 intergenic spacers that were common to all three species. Of these, five spacers fulfilled our selection criteria and were named MST2, MST3, MST5, MST6 and MST7. Use of the PCR primers listed in Table 1 to amplify each of the five spacers produced amplicons ranged from 333 -bp to 738-bp and sequence reads ranging from 246-bp to 543bp (Table 1).

\section{Interspecies analysis}

Pairwise comparison of the five spacers (Table 2 and figure 1) revealed they had species-specific sequence with interspecies sequence differences relying on single nucleotide polymorphism in $36(90 \%)$ cases, deletion in $3(7.5 \%)$ cases and insertion in 1 (2.5\%) case. Comparing B. duttonii MST7 with B. crocidurae and B. recurrentis MST7 yielded $93 \%$ and $97 \%$ similarity, respectively, whilst comparing $B$. crocidurae MST7 with B. recurrentis MST7 showed $93 \%$ similarity. The other four spacers yielded pairwise sequence similarity of $97-99 \%$ (Table 2). Sequences for each allele of each spacer have been deposited in GenBank under accession number (JQ398815: JQ398841) as well as in our local data base (http://www.ifr48.com).

\section{Intra-species analysis}

While the concatenation of the five spacers yielded a discrimination index of $0.825,1$, this index was of 0.7814 for MST2, 0.6896 for MST6, 0.6749 for MST5, 0.6623 for MST7, and 0.6579 for MST3. Concatenation of the five spacers yielded 8

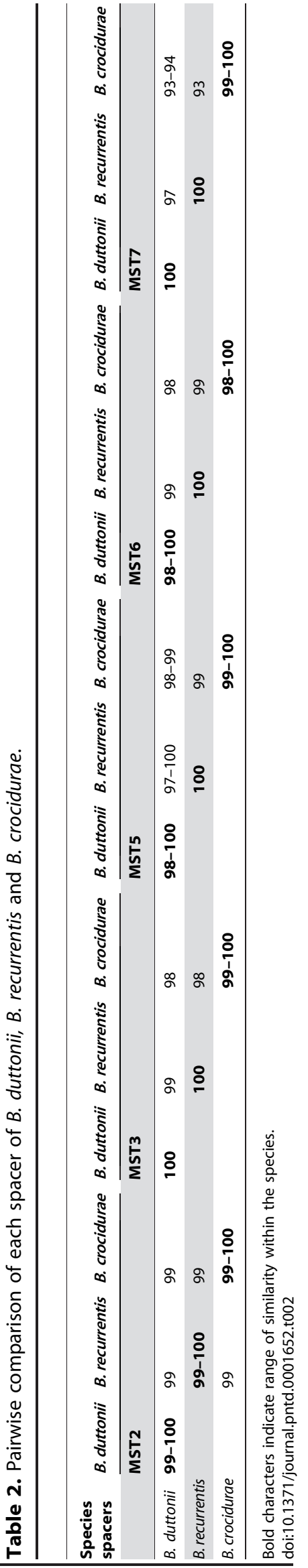




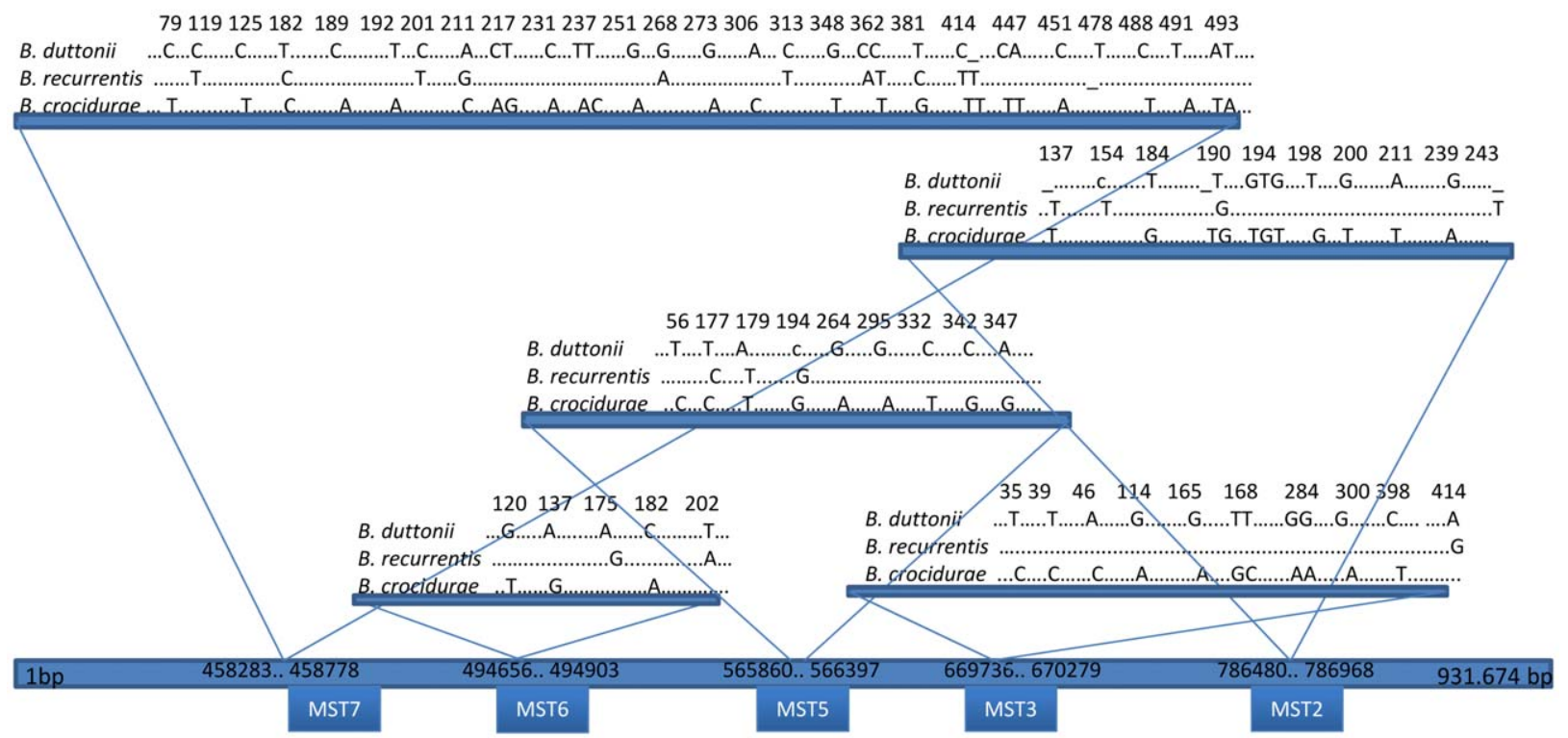

Figure 1. Distribution of spacers among the chromosome of $B$. duttonii and main differences within each spacer. doi:10.1371/journal.pntd.0001652.g001

STs named ST6-ST13 for the 13 B. crocidurae samples and the $B$. crocidurae Achema type strain (Table 3; Figure 2). 3 STs named ST1-ST3 for the 18 B. duttonii samples and the B. duttonii Ly type strain and 2 STs named ST4-ST5 for the 30 B. recurrentis samples and the $B$. recurrentis A1 type strain. MST2 sequencing classified latter samples into ST-4 (11 samples) and ST-5 (19 samples) due to the insertion of a $\mathrm{G}$ at position 190. The genotype ST-5 represented $47.6 \%$ (10 out 21 samples) detected in 1994 and all the nine samples detected in 2011 .

\section{MST-based phylogenetic analysis of RF Borrelia}

The phylogenetic tree constructed after concatenation of the five intergenic spacer sequences separated the RF Borrelia into three clades, each clade containing only one Borrelia species (figure 2). A first clade comprised of all the 30 B. recurrentis isolates; a second clade comprised of three groups representing the three $B$. duttonii spacer types and a last clade comprised of 7 B. crocidurae spacer types.

\section{Discussion}

PCR-derived data reported herein were interpreted as authentic as the negative controls used in every PCR-based experiment remained negative, all the PCR products were sequenced and experiments yielded reproducible sequences. We therefore established the proof-of-concept that MST could be used for species identification and genotyping of 3 out of 4 cultured RF borreliae (B. hispanica was not available for this study) in Africa. MST combines the sensitivity of PCR with unambiguous, portable data yielded by sequencing. Indeed, all the sequences determined are freely available in GenBank and in our local database website at

Table 3. List of spacer types (ST) found in this study.

\begin{tabular}{|c|c|c|c|c|c|c|c|}
\hline Species & Strains & ST & MST2 & MST3 & MST5 & MST6 & MST7 \\
\hline \multirow[t]{3}{*}{ B. duttonii } & $\mathrm{Bd} 9,11,17$ & 1 & 1 & 1 & 1 & 1 & 1 \\
\hline & Bd $1,2,3,5,6,8,12,13,16,19,20,22$, Ly & 2 & 2 & 1 & 2 & 2 & 1 \\
\hline & Bd15, 18 & 3 & 2 & 1 & 3 & 2 & 1 \\
\hline \multirow[t]{2}{*}{ B. recurrentis } & $\operatorname{Br} 1,2,3,4,5,6,7,8,9,10,11$ & 4 & 3 & 2 & 4 & 3 & 2 \\
\hline & $\begin{array}{l}\mathrm{Br} 12,13,14,15,16,17,18,19 \\
20,21,22,23,24,25,26,27,28,29,30, \mathrm{~A} 1\end{array}$ & 5 & 4 & 2 & 4 & 3 & 2 \\
\hline \multirow[t]{8}{*}{ B. crocidurae } & B.cr18, B.cr89, B.cr88 B.cr85 & 6 & 5 & 3 & 5 & 7 & 4 \\
\hline & B.cr34 & 7 & 5 & 4 & 5 & 7 & 5 \\
\hline & B.cr30 & 8 & 6 & 4 & 5 & 4 & 4 \\
\hline & B.cr35 & 9 & 5 & 4 & 5 & 5 & 3 \\
\hline & B.cr936 & 10 & 5 & 3 & 5 & 6 & 5 \\
\hline & B.cr81 B.cr57 B.cr40, B.cr23 & 11 & 5 & 4 & 5 & 6 & 5 \\
\hline & B.cr66 & 12 & 5 & 4 & 5 & 7 & 4 \\
\hline & Achema & 13 & 7 & 5 & 6 & 8 & 6 \\
\hline
\end{tabular}

doi:10.1371/journal.pntd.0001652.t003 


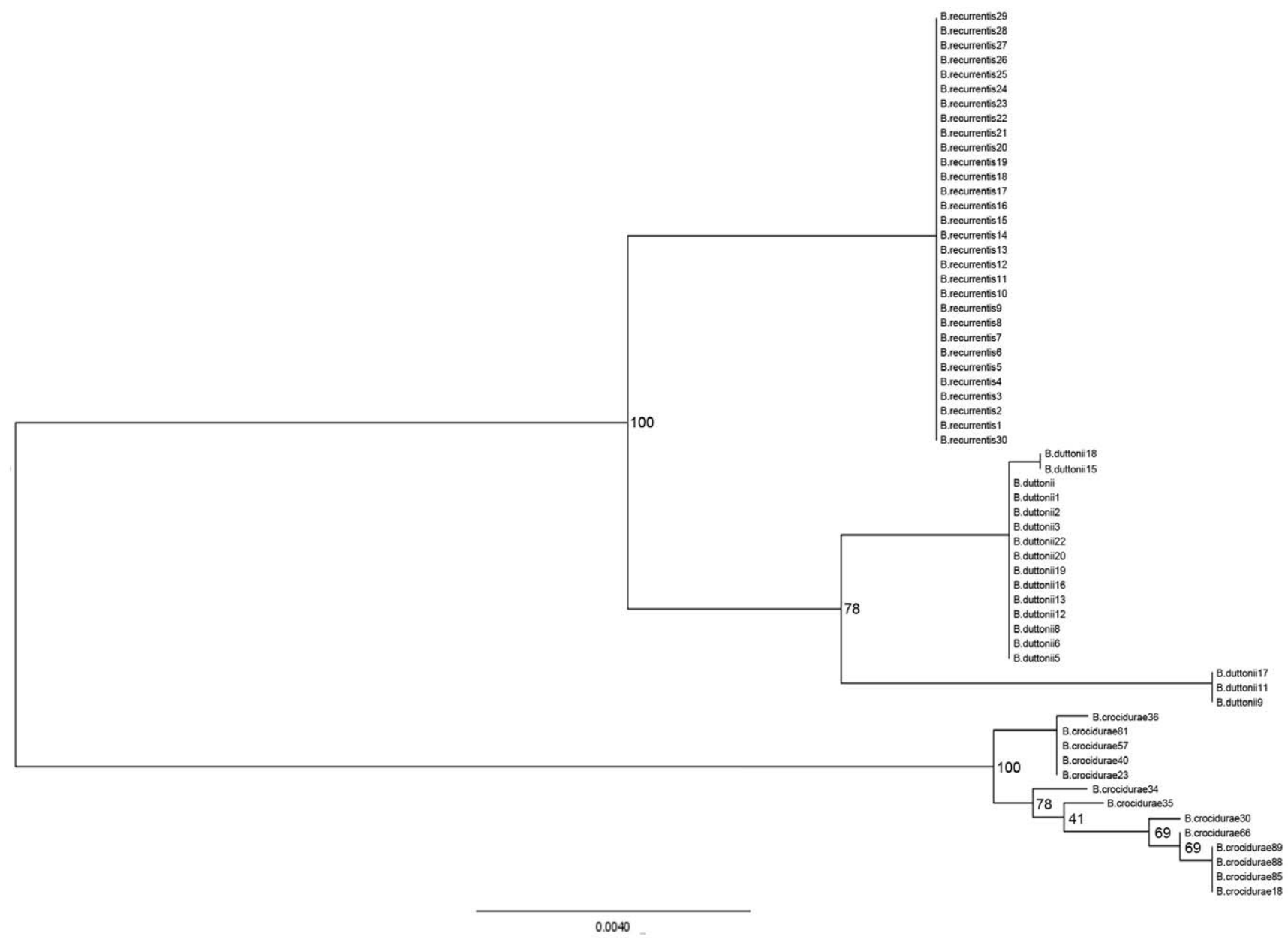

Figure 2. Maximum-likelihood tree based on five intergenic spacers sequences for $\mathbf{6 1}$ Borrelia strains. To examine the confidence of ML tree, 100 bootstrap replicates were used. doi:10.1371/journal.pntd.0001652.g002

ifr48.com. Therefore, any laboratory with a capacity in PCRsequencing could easily confirm and compare their data with that reported herein to further increase the knowledge of RF Borrelia species and genotypes circulating in African countries.

In the present study, five intergenic spacers were selected from the alignment of $B$. crocidurae, $B$. duttonii and B. recurrentis reference genomes, representing approximately $\sim 0.2 \%$ of the total genome length. The spacers were scattered across the chromosome thus representative of the whole genome. Such a multi-target approach offers distinct advantages over the one single locus methods previously used, such as the 16S-23S IGS for typing that may be less representative of the whole genome. Based on this spacer sequencing, a total of $61 \mathrm{RF}$ strains could be separated into 12 STs. Interestingly, we observed that isolates grouped into three clades corresponding to the three Borrelia organisms under study. Indeed, MST yielded no overlap between $B$. duttonii and $B$. recurrentis organisms contrary to that observed when using IGS typing $[1,7]$. We observed that sequencing MST7 spacer alone accurately discriminated between $B$. duttonii and $B$. recurrentis with $3 \%$ sequence divergence, a result not previously achieved. Therefore, sequencing MST7 spacer alone could be used for the molecular identification of RF Borrelia in Africa at the species level, but not for genotyping which requires sequencing the four other spacers in addition to MST7.
Further analysis indicated that each one of the three Borrelia species under study was comprised of several spacer-types. $B$. recurrentis was the least diverse Borrelia comprising of only two very closely related groups. This finding supports the previous genomic analysis that concluded that $B$. recurrentis was a subset of $B$. duttonii [16]. In our study also, there was an inverse correlation between the RF Borrelia MST diversity and the reported mortality rate for these RF Borrelia [8,15].

Despite the fact that we tested a small set of $B$. crocidurae, nevertheless we found a high diversity index in this species since 13 B. crocidurae samples collected in Senegal yielded 7 MST types and the $B$. crocidurae Achema type strain collected in Mauritania yielded an additional MST type. This first genotyping method for $B$. crocidurae is therefore very promising to probe its geographic repartition as well as potential association of $B$. crocidurae genotypes with vectors. Indeed, four genogroups could be identified in $O$. sonrai ticks collected in Senegal and Mauritania [24]. In this study, $B$. crocidurae flagellin sequence was found identical among the four $O$. sonrai tick groups but the $B$. crocidurae infection rate significantly differed among the four tick groups; MST may help studying such discrepancy and may reveal previously unknown relationships between $B$. crocidurae genotypes and $O$. sonrai genotypes. Moreover, a recent study indicated that $B$. crocidurae may be transmitted by soft tick Ormithodoros erraticus in Tunisia, challenging $O$. sonrai as the 
only $B$. crocidurae vector in West Africa [25]. MST is new laboratory tool to question whether the unexpected higher diversity in $B$. crocidurae than in $B$. duttonii and $B$. recurrentis is linked to a more complex cycle involving several mammals and ticks species.

Present data indicate that MST is offering a new sequencingbased technique for further exploring the identification and genotypes of RF Borrelia in vectors and clinical specimens collected in Africa.

\section{References}

1. Scott J, Wright D, Cutler S (2005) Typing African relapsing fever spirochetes. Emerg Infect Dis 11: 1722-1729.

2. Ras NM, Lascola B, Postic D, Cutler SJ, Rodhain F, et al. (1996) Phylogenesis of relapsing fever Borrelia spp. Int J Syst Bacteriol 46: 859-865.

3. Houhamdi L, Raoult D (2005) Excretion of living Borrelia recurrentis in feces of Infected Human Body Lice. J Infect Dis 191: 1898-1906.

4. Kisinza WN, McCall PJ, Mitani H, Talbert A, Fukunaga M (2003) A newly identified tick-borne Borrelia species and relapsing fever in Tanzania. Lancet 18: 1283-1284.

5. Sarih M, Garnier M, Boudebouch N, Bouattour A, Rihani A, et al. (2009) Borrelia hispanica relapsing fever, Morocco. Emerg Infect Dis 15: 1626-1629.

6. Parola P, Diatta G, Socolovschi C, Mediannikov O, Tall A, et al. (2011) Tickborne relapsing fever borreliosis, rural Senegal. Emerg Infect Dis 17: 883-885.

7. Cutler SJ, Bonilla EM, Singh RJ (2010) Population structure of East African relapsing fever Borrelia spp. Emerg Infect Dis 16: 1076-1080.

8. Borgnolo G, Denku B, Chiabrera F, Hailu B (1993) Louse-borne relapsing fever in Ethiopian children: a clinical study. Ann Trop Paediatr 13: 165-171.

9. Nordstrand A, Bunikis I, Larsson C, Tsogbe K, Schwan TG, et al. (2007) Tickborne relapsing fever diagnosis obscured by malaria, Togo. Emerg Infect Dis 13: 117-123.

10. Vial L, Diatta G, Tall A, Ba el H, Bouganali H, et al. (2006) Incidence of tickborne relapsing fever in west Africa: longitudinal study. Lancet 368: 37-43.

11. Ramos JM, Reyes F, Tesfamariam A, Malmierca E (2007) Louse-borne relapsing fever and malaria co-infection in Ethiopia. Trop Doct 37: 121-122.

12. Miron D, Olshinsky A, Assy N, Zuker M, Efrat M, et al. (2004) Plasmodium and Borrelia co-infection. J Travel Med 11: 115-116.

13. Jongen VH, van Roosmalen J, Tiems J, Van Holten J, Wetsteyn JC (1997) Tickborne relapsing fever and pregnancy outcome in rural Tanzania. Acta Obstet Gynecol Scand. pp 834-839.

14. Dupont HT, La Scola B, Williams R, Raoult D (1997) A focus of tick-borne relapsing fever in southern Zaire. Clin Infect Dis. pp 139-144.

\section{Author Contributions}

Conceived and designed the experiments: DR MD. Performed the experiments: EH. Analyzed the data: EH GG MD SGB SJC DR. Contributed reagents/materials/analysis tools: CS KDB JA SCB SJC. Wrote the paper: DR MD EH.

15. Ramos J, Malmierca E, Reyes F, Wolde W, Galata A, et al. (2004) Characteristics of louse-borne relapsing fever in Ethiopian children and adults. Ann Trop Med Parasitol 98: 191-196.

16. Lescot M, Audic S, Robert C, Nguyen TT, Blanc G, et al. (2008) The genome of Borrelia recurrentis, the agent of deadly louse-borne relapsing fever, is a degraded subset of tick-borne Borrelia duttonii. PLoS Genet 12: e1000185.

17. Toledo A, Anda P, Escudero R, Larsson C, Bergstrom S, et al. (2010) Phylogenetic analysis of a virulent Borrelia species isolated from patients with relapsing fever. J Clin Microbiol 48: 2484-2489.

18. Drancourt M, Roux V, Dang LV, Lam THCD, Chenal-Francisque V, et al. (2004) Genotyping, Orientalis-like Yersinia pestis, and plague pandemics. Emerg Infect Dis 10: 1585-1592.

19. Zhu Y, Fournier PE, Ogata H, Raoult D (2005) Multispacer typing of Rickettsia prowazekii enabling epidemiological studies of epidemic typhus. J Clin Microbiol 43: 4708-4712.

20. Ning Z, Cox AJ, Mullikin JC (2001) SSAHA: a fast search method for large DNA databases. Genome Res 11: 1725-1729.

21. Corpet $\mathrm{F}$ (1988) Multiple sequence alignment with hierarchical clustering. Nucl Acids Res 16: 10881-10890.

22. Hunter PR, Gaston MA (1988) Numerical index of the discriminatory ability of typing systems: an application of Simpson's index of diversity. J Clin Microbiol 26: 2465-2466.

23. Guindon S, Dufayard JF, Lefort V, Anisimova M, Hordijk W, et al. (2010) New algorithms and methods to estimate maximum-likelihood phylogenies: assessing the performance of PhyML 3.0. Syst Biol 59: 307-321.

24. Vial L, Durand P, Arnathau C, Halos L, Diatta G, et al. (2006) Molecular divergences of the Ornithodoros sonrai soft tick species, a vector of human relapsing fever in West Africa. Microbes Infect 8: 2605-2611.

25. Bouattour A, Garnier M, M'Ghirbi Y, Sarih M, Gern L, et al. (2010) Borrelia crocidurae infection of Ormithodoros erraticus (Lucas, 1849) ticks in Tunisia. Vector Borne Zoonotic Dis 10: 825-830. 